\title{
ReaR

\section{Metaanálisis entre la videolaringoscopia y la laringoscopia directa en el manejo de la vía aérea difícil por parte de anestesiólogos experimentados}

Artículo original: Videolaryngoscopy vs. direct laryngoscopy use by experienced anaesthetists in patients with known difficult airways: a systematic review and meta-analysis. Pieters BMA, Maas EHA, Knape JTA, Van Zundert AAJ. Anaesthesia. 2017 Dec;72(12):1532-1541. (PubMed)

Zugasti Echarte $O$.

\section{Complejo Hospitalario de Navarra.}

\section{Resumen}

En este artículo se presenta una revisión sistemática y metaanálisis para determinar si la literatura indicaba que los videolaringoscopios suponen una ventaja sobre la laringoscopia directa cuando son utilizados por anestesiólogos experimentados en el manejo de la vía aérea difícil prevista.

El éxito en la intubación al primer intento fue mayor en el grupo de los videolaringoscopios.

Su uso también se asoció con una significativa mejor visión de la glotis y con un menor traumatismo de la mucosa de la vía aérea.

Se concluye que la videolaringoscopia es un valor añadido para el anestesiólogo experimentado, mejorando la intubación en el primer intento, la visión de la glotis y reduciendo el trauma de la mucosa, pudiendo tener un papel importante en el abordaje inicial de la vía aérea difícil prevista.

\section{Introducción}

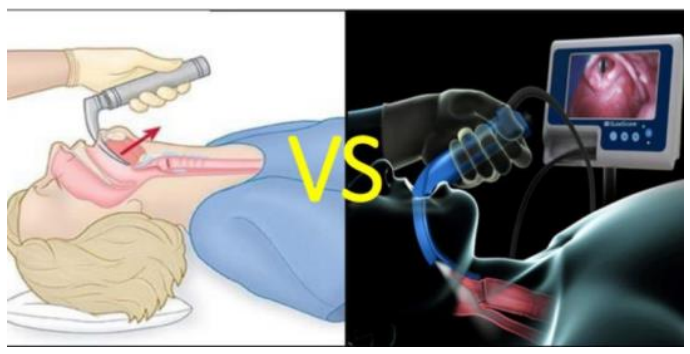

En este artículo se presenta una revisión sistemática y metaanálisis para determinar si la literatura indicaba que los videolaringoscopios suponen una ventaja sobre la laringoscopia directa cuando son utilizados por anestesiólogos experimentados en el manejo de la vía aérea difícil prevista.

El éxito en la intubación al primer intento fue mayor en el grupo de los videolaringoscopios.
$\mathrm{Su}$ uso también se asoció con una significativa mejor visión de la glotis y con un menor traumatismo de la mucosa de la vía aérea.

Se concluye que la videolaringoscopia es un valor añadido para el anestesiólogo experimentado, mejorando la intubación en el primer intento, la visión de la glotis y reduciendo el trauma de la mucosa, pudiendo tener un papel importante en el abordaje inicial de la vía aérea difícil prevista.

En el manejo de la vía aérea, la laringoscopia directa (LD) es probablemente la técnica utilizada de entrada en la mayoría de las intubaciones traqueales. No obstante, la intubación dificultosa, fallida o prolongada, así como los escenarios de "no intubable-no ventilable" o " no 
intubable-no oxigenable" todavía representan el $39 \%$ de todos los eventos ocurridos durante la anestesia (1).

Los videolaringoscopios (VL) suponen una nueva herramienta para afrontar la vía aérea difícil. Sin embargo, su utilización no es garantía de éxito. El éxito en la intubación depende de múltiples factores, como son: el diseño (angulación, tipo de pala, disponibilidad de canal), la calidad de la imagen en el monitor, la iluminación de la laringe $u$ orofaringe, la anatomía del paciente, la historia previa de intubación difícil, la intubación electiva o de emergencia, la experiencia y la habilidad del intubador y el lugar donde la intubación se lleva a cabo.

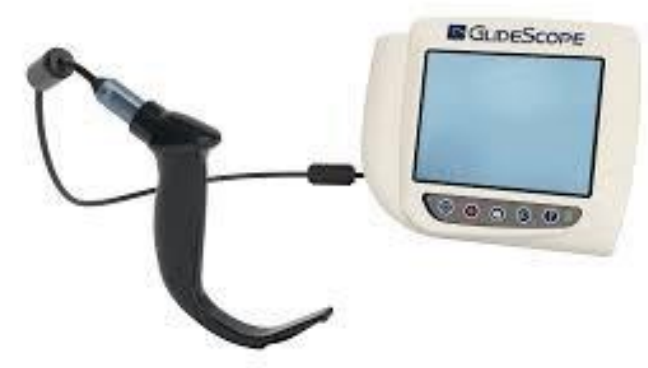

Imagen 1. VL Glidescope (www.chsltd.com)

La experiencia del anestesiólogo es un factor que no puede ser subestimado. La videolaringoscopia tiene una curva de aprendizaje rápida (2), pero debe ser enseñada y no deben emplearse por primera vez estos dispositivos al enfrentarse a un paciente difícil de intubar. Una desventaja, especialmente para anestesiólogos experimentados, relativamente inexpertos con la videolaringoscopia, puede ser la angulación aguda de la pala curva de algunos dispositivos, como el VL Glidescope (Imagen 1) o el McGrath (Imagen 2).

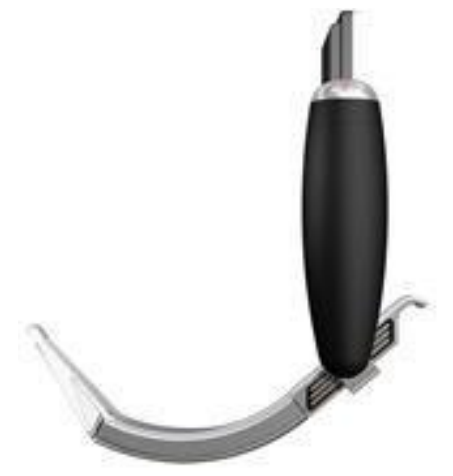

Imagen 2.
(www.keywordsugge.com) $\quad$ VL McGrath

El anestesiólogo familiarizado con la laringoscopia directa, intentará inconscientemente alinear los ejes oral, faríngeo y traqueal, lo que puede obstaculizar la visión cuando se utiliza un VL con pala con ángulo agudo.

En la experiencia clínica, la mejoría de la visión laríngea no siempre conduce a una intubación más fácil o exitosa. Dos preguntas pertinentes son: ¿es la videolaringoscopia superior a la laringoscopia directa en pacientes con una vía aérea difícil conocida cuando es utilizada por anestesiólogos con experiencia con la laringoscopia directa? Y, de ser así, ¿debería abandonarse completamente la laringoscopia directa para la intubación de pacientes con vía aérea difícil conocida y convertirse la videolaringoscopia en el nuevo estándar?

La intención de los autores fue realizar una revisión sistemática y metaanálisis para comprobar si la literatura apoya el uso de VL por parte de anestesiólogos experimentados para la intubación de pacientes con vía aérea difícil conocida.

\section{Material y Métodos}

Se realizaron búsquedas en PubMed, MEDLINE, Embase y el registro central de ensayos clínicos de Cochrane hasta enero de 2017. En el protocolo se 
incluyeron ensayos clínicos que compararan laringoscopios clásicos con videolaringoscopios angulados.

Los criterios de inclusión fueron: aleatorización, estudios controlados, observacionales y estudios de cohortes, población de pacientes mayor de 18 años, vía aérea difícil sospechada, cirugía electiva, intubación traqueal realizada por anestesiólogos con más de dos años de experiencia con LD y estudios en los que se comparase LD con VL en el mismo paciente.

Los resultados extraídos de los estudios que se estudiaron fueron: éxito al primer intento en la intubación traqueal, tiempo hasta la intubación exitosa, número de intentos de intubación, grado de Cormack-Lehane, utilización de complementos a la intubación (estiletes, guías) y complicaciones (trauma dental o de la mucosa).

Todos los criterios de inclusión se cumplieron por nueve estudios, incluyendo 1329 pacientes. Los VL utilizados fueron Glidescope, C-MAC (Imagen 3), C-MAC D-Blade (Imagen 4), Pentax AWS (Imagen 5), Airtraq (Imagen 6) y McGrath.

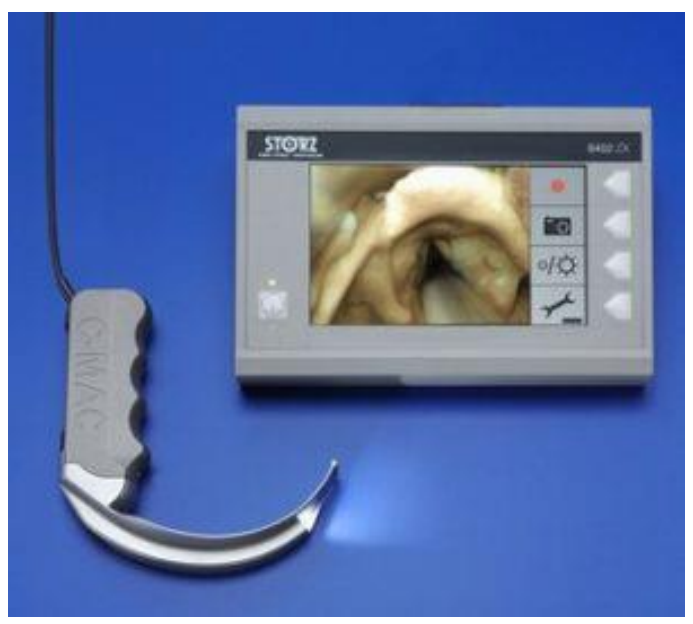

$\begin{array}{lcrl}\text { Imagen } & 3 . & \text { VL } & \text { C-MAC } \\ \text { (http://www.sciencedirec.com) }\end{array}$
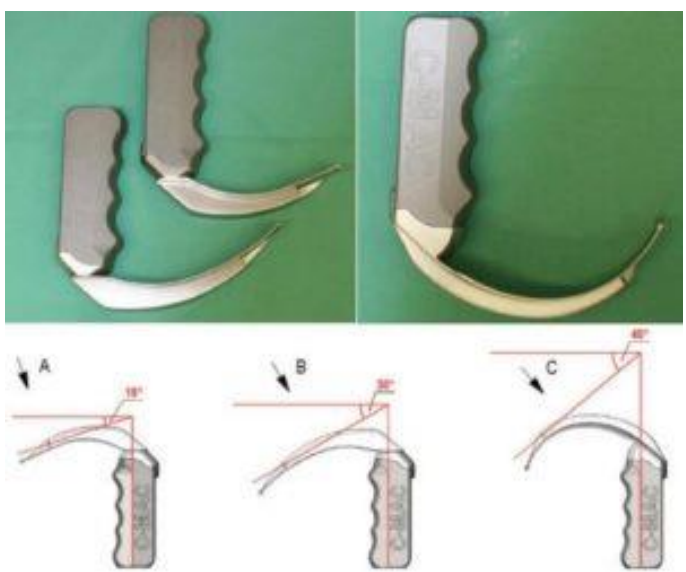

Imagen

4.

Pala

D-Blade

(http://www.picquery.com)

Los datos de los estudios incluidos se combinaron para calcular el efecto acumulado de los videolaringoscopios frente a la laringoscopia directa. Para todos los análisis se utilizó Review Manager, versión 5.3 descargado el 11 de enero de 2017.

Para este metaanálisis, se utilizó el modelo de efectos aleatorios. La heterogeneidad entre ensayos se cuantificó con la $\mathrm{I}^{2}$ de Higgins y Thompson. La $\mathrm{I}^{2}$ describe el porcentaje de variación total entre los estudios que es debido a la heterogeneidad más que al azar. Un valor del $0 \%$ indica que no se ha observado heterogeneidad, mientras que valores mayores indican heterogeneidad creciente. Un $\mathrm{I}^{2}>50 \%$ se considera que representa una heterogeneidad sustancial.

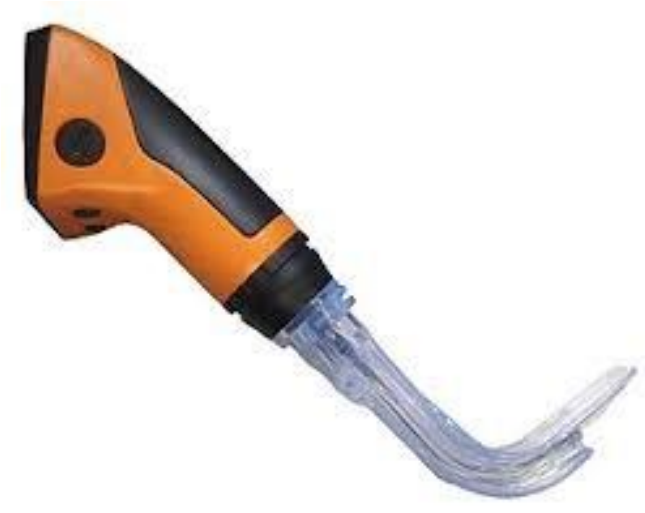

Imagen 5. VL Pentax AWS (http://www.richardsmedical.com) 


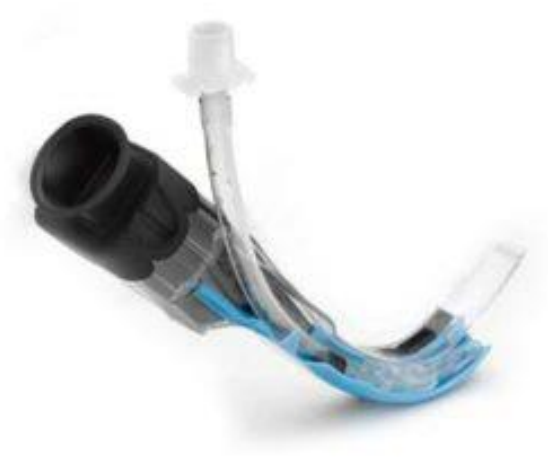
Imagen $\quad 6$.
$(\underline{\text { http://www.airtraq.com/es) }}$

\section{Resultados}

- La elección de utilizar VL o LD influyó en el éxito en la intubación al primer intento, que fue mayor con todos los videolaringoscopios $\quad(95 \%$ vs $86 \%$ ), con significación estadística.

- La media (IC 95\%) de tiempo utilizado para la intubación exitosa fue de $28 \mathrm{~s}$ para los VL en comparación con los $37 \mathrm{~s}$ de la LD. Se repitió el análisis incluyendo únicamente los estudios en los que se había empleado el GlideScope, mostrando una media (IC 95\%) de tiempo utilizado para la intubación exitosa de $30 \mathrm{~s}$ en comparación con los $42 \quad \mathrm{~s}$ empleados en el grupo de laringoscopia directa. Aparece un alto nivel de heterogeneidad $\left(\mathrm{I}^{2} 97 \%\right)$, que puede ser explicado por las diferentes maneras en los que los distintos estudios midieron el tiempo hasta la intubación.

- Para la evaluación del grado de Cormack-Lehane (CL), se hicieron 2 grupos: CL grados $1 \mathrm{y}$ 2 vs CL grados 3 y 4 . En el grupo de VL se registraron más grados CL 1 y 2 (96\%) que cuando se utilizó la LD (34\%). Con LD un $35.7 \%$ resultaron ser un $\mathrm{CL}>3$, mientras que con $\mathrm{VL}$ esto únicamente ocurrió en un $3 \%$. El grado de CL fue medido de diferentes maneras por los distintos investigadores, lo que resulta en un alto nivel de heterogeneidad estadística $\left(\mathrm{I}^{2} 88 \%\right)$.

- Sólo cuatro estudios recogieron el uso de complementos como ayuda a la intubación. Estos fueron: estilete, guía elástica, guía semi flexible, manipulación laríngea externa y cambios en la colocación de la cabeza. No se encontraron diferencias en el uso de complementos a la intubación entre las dos técnicas.

- El número de intentos de intubación fue recogido en 5 estudios. Los participantes necesitaron más de un intento cuando utilizaban LD en el $10.3 \%$ de los casos frente al $9 \%$ con VL. Los intentos se definieron y se registraron de distinta forma en los diferentes estudios, lo que resulta en un alto nivel de heterogeneidad estadística $\left(\mathrm{I}^{2} 73 \%\right)$.

- En tres de los estudios se notificó traumatismo dental, que fue mayor con LD. La definición de lo que se considera una complicación dental y la forma en la que se registró fueron muy diferentes entre los estudios, por lo que existe una gran heterogeneidad estadística $\left(I^{2} 63 \%\right)$.

- Respecto al traumatismo de la mucosa, se registró una incidencia del $2 \%$ con los VL y de un $12 \%$ con $L D$, siendo esta diferencia estadísticamente significativa.

\section{Conclusiones}

- La conclusión principal es que la videolaringoscopia se relaciona con un mayor éxito en la 
intubación al primer intento, incluso para los anestesiólogos experimentados con la laringoscopia directa.

- En este estudio el uso de VL no se vio asociado a una reducción significativa del tiempo hasta lograr una intubación exitosa.

- En este metaanálisis la videolaringoscopia proporcionó una mejor visión de la glotis. El número de laringoscopias de grado 3 o 4 en la escala CL fue menor en general en el grupo de VL y en particular con los videolaringoscopios con pala angulada (ej. Pentax AWS, Airtraq, GlideScope y C-MAC D-Blade).

- Los videolaringoscopios son un valor añadido para el anestesiólogo con experiencia.

\section{Comentario}

- La diferencia encontrada entre los grupos respecto a la intubación al primer intento fue del $9 \%$, a favor del grupo de los VL. Aunque no parezca una gran diferencia, hay que recordar que el riesgo de complicaciones aumenta conforme más intentos de intubación se realizan. Repetir el intento de intubación aumenta el riesgo de desembocar en una situación de "no intubable no ventilable" y el American Society of Anesthesiologist Closed Claims Proyect (ASACCP) (3) sugiere un aumento de las tasas de muerte y daño cerebral asociados a estas situaciones. Cuando la LD no es exitosa, los siguientes intentos con la misma técnica tienen una tasa de fallo próxima al $80 \%$, mientras que la utilización de técnicas alternativas es más eficiente. Las guías de la
Difficult Airway Society recalcan que es esencial optimizar la tasa de éxito en la intubación al primer intento (1). Por tanto, reservar los videolaringoscopios para un segundo intento puede malgastar el primero y hacer el segundo más dificultoso.

- Esta revisión subraya el valor añadido que supone la videolaringoscopia para los expertos en laringoscopia directa, tanto que debería formar parte del manejo estandarizado de la vía aérea difícil y tal vez incluso ser el abordaje inicial de la intubación en estas circunstancias.

- Los hospitales deben proporcionar un arsenal de VL que permita a los anestesiólogos la opción de elegir el más adecuado. El rango de disponibilidad debe ser restringido por razones de económicas, pero los departamentos de anestesia deben tener en cuenta las recomendaciones de las guías clínicas.

- Los VL con pala de ángulo agudo requieren una técnica diferente de intubación, ya que la forma de la pala sigue la anatomía natural de la cavidad oral y la cámara en la punta de la pala sitúa el punto de visión del operador en una posición muy próxima a la glotis.

- Con la videolaringoscopia pese a tener una visión perfecta de la glotis, puede haber todavía dificultades para colocar el tubo endotraqueal en posición correcta. Este problema puede resolverse fácilmente con el uso de un estilete, como recomiendan algunos fabricantes. El uso generalizado de estiletes puede parecer un 
tema de importancia menor, aunque debe tenerse en cuenta que el empleo de estiletes rígidos aumenta el riesgo de sufrir traumatismo en la mucosa, sobre todo cuando el VL es un dispositivo voluminoso y deja poco espacio para maniobrar (4).

- La experiencia con la laringoscopia directa no es equiparable a la habilidad con la videolaringoscopia y el ser experto en el uso de un VL no le hace a uno automáticamente habilidoso con todos los dispositivos. Cortellazzi et al. mostraron en su estudio que la videolaringoscopia es una técnica compleja que requiere una práctica intensiva para adquirir experiencia, incluso en aquellos profesionales entrenados en la laringoscopia directa (2).

- Esta revisión sistemática y metaanálisis tiene limitaciones.

- En los estudios originales, los anestesiólogos no eran ciegos al dispositivo utilizado, lo que puede haber conducido a un resultado alterado debido al efecto Hawthorne (los individuos saben que están siendo estudiados y cambian su comportamiento).

- El análisis del tiempo hasta que se consiguió la intubación exitosa fue complicado, ya que los diferentes estudios utilizaban distintas definiciones para el comienzo y el final de la medida del tiempo empleado para la intubación lo que resulta en un nivel extremadamente alto de heterogeneidad.

- El comportamiento de los VL depende de las circunstancias exactas de la vía aérea difícil y el dispositivo óptimo puede variar entre diferentes circunstancias de vía aérea difícil.

- El sesgo de publicación puede estar presente, lo que podría significar que estudios más pequeños que favorezcan a la LD no hayan sido publicados.

- Las futuras

investigaciones deberían enfocarse en comparar el amplio arsenal de VL disponible, de tal manera que puedan emitirse recomendaciones sobre qué dispositivo debe usarse para cada paciente, teniendo en cuenta las características del paciente y la experiencia del operador del VL.

\section{Bibliografía}

1. Cook TM, Woodall N, Frerk C. Major complications of airway management in the UK: results of the 4th National Audit Project of the Royal College of Anaesthetists and the Difficult Airway Society. Part 1 Anaesthesia. British Journal of Anaesthesia 2011; 106: 617-31. (ubMed) (NML) ( $\underline{\text { PDF }})$

2. Cortellazzi P, Caldiroli D, Byrne A, Sommariva A, Orena EF, Tramacere I. Defining and developing expertise in tracheal intubation using a GlideScope for anaesthetists with expertise in Macintosh direct laryngoscopy: an invivo longitudinal study. Anaesthesia 2015; 70: 290-5. ( PubMed) (HTML) (PDF)

3. Peterson GN, Domino KB, Caplan RA, Posner KL, Lee LA, Cheney FW. Management of the difficult airway: a 
closed claims analysis. Anesthesiology 2005; 103: 33-9. ( PubMed) (HTML)

4. Marson BA, Anderson E, Wilkes AR, Hodzovic I. Bougie-related airway trauma: dangers of the hold-up sign. Anaesthesia. 2014 Mar; 69 (3): 219-23. (PubMed) (HTML) (ePDF)
Correspondencia al autor

Orreaga Zugasti Echarte orreaga.zugasti.echarte@.cfnavarra.es F.E.A. Anestesiología y Reanimación. Complejo Hospitalario de Navarra.

Aceptado para blog en marzo de 2018. 